\title{
Sharing animals across protocols
}

Dr. Larry Chen was the Principal Investigator (PI) on an IACUC protocol which involved performing an orthopedic surgical procedure using a rabbit model. Chen's colleague, Dr. Riva Rosen, was the PI on a study involving long bone fracture healing, using a mouse model. About two years earlier, when Chen's protocol was first approved, Rosen had worked with Chen and she had performed the same orthopedic surgery on rabbits that was now being performed only by Chen. Both researchers were board-certified orthopedic surgeons and their research was funded through $\mathrm{NIH}$ grants. Over time it became clear to Rosen that she would need to use an animal much larger than a mouse for a proof-of-concept study that she wanted to perform, and the procedure used by Chen on rabbits precisely met her research needs. So Rosen 'borrowed' two of Chen's rabbits and performed the exact same procedure used by Chen, without any complications.

Neither Rosen nor Chen thought they were doing anything wrong until the IACUC found out what had happened and told Rosen that she did not have IACUC approval to use Chen's animals or perform the surgical procedure. Chen was told that he did not have IACUC approval to give the rabbits to Rosen. In her own defense, Rosen said that the surgery she performed on the rabbits was already approved by the IACUC and that she herself was approved by the IACUC to do the surgery. Chen said nearly the same thing but added that after the IACUC contacted him he looked at the PHS Policy on Humane Care and Use of Laboratory Animals ${ }^{1}$ and at the Animal Welfare Act regulations ${ }^{2}$, and both documents said that the IACUC had to approve activities related to the care and use of animals. Chen's point, like Rosen's, was that the use of rabbits for the surgery had been reviewed and approved by the IACUC.

Chen and Rosen have argued that Rosen's so-called unapproved surgical work was actually approved by the IACUC. Chen did not find anything in the documents he consulted to indicate that the surgery had to be performed under a single IACUC approved protocol. The two researchers believed that as long as all the component parts of a study were approved, the research could go forward without additional approvals. Do you agree with Chen and Rosen? How would you approach this problem?

1. Public Health Service. Policy on Humane Care and Use of Laboratory Animals. (US Department of Health and Human Services, Washington, DC, 1986; amended 2002).

2. Animal Welfare Act Regulations. 9 CFR. Chapter 1, Subchapter A, Part 2, Subpart C.

\section{RESPONSE}

\section{'Borrowing' is not an acceptable practice}

\author{
Adrienne Ferguson Duran, BAS, LVT, \\ RLATG, CMAR \& \\ Peggy T. Tinkey, DVM, DACLAM
}

Rosen was not in compliance when she 'borrowed' the two rabbits for several reasons.

Rosen was doing a proof-of-concept or pilot study. Even pilot studies must be reviewed and approved by the IACUC, and in this case, the IACUC did not have a chance to review the scientific aims of Rosen's study. Even though the surgery might have been identical to the surgery in Chen's protocol, IACUC approvals must be project-specific ${ }^{1}$. In this case, the scientific aims could have been very different, and even if the surgical technique, personnel, and training were already approved by the IACUC, the IACUC could have opted not to approve the use of rabbits for protocolspecific reasons. If experimental goals are different then veterinary intervention and enrichment might also need to be different. For example, with different scientific aims, the use of antibiotics or pair housing that might be acceptable for one PI and protocol might not be appropriate for the other PI and protocol. In addition to the scientific objectives and procedures, the IACUC needs an opportunity to review the statistical justification for the species and number of animals ${ }^{2}$. For instance, do two rabbits provide enough statistical power for even a proof of concept? Is this a duplication of Chen's previous work?

In this scenario, it sounds like the anesthesia, staff training and analgesia were all appropriate, but the IACUC should also have the opportunity to review and approve those items again for the pilot study. Depending on the scope of this pilot study, the IACUC may allow an amendment to Chen's protocol. Most PIs would find this more desirable than submitting a new protocol.
Animals cannot be 'borrowed' to carry out pilot studies or experiments with research goals that differ from those of the originally approved protocol. They must be transferred appropriately from one study's proposal to another to verify that the new proposal has been approved. In addition, animals should be transferred across protocols to allow for proper identification of animal subjects. The Guide for the Care and Use of Laboratory Animals states that identification cards should include several key pieces of information, including the PI's name and protocol ${ }^{2}$. If the animals are not transferred through the proper channels they will not be labeled with the correct information. In this case, the animals would have been identified with Chen's name and protocol number and not Rosen's. It is also inappropriate to 'borrow' animals because the IACUC must ensure that the same animals are not being used for multiple invasive or surgical procedures. In this scenario, it is unclear whether the animals that Rosen borrowed were naive or not. If the animal 